\title{
Early Detection of Cystic Fibrosis Acute Pulmonary Exacerbations by Exhaled Breath Condensate Metabolomics
}

\author{
Xiaoling Zang, ${ }^{\dagger}$ María Eugenia Monge, ${ }^{\dagger}$ (৫) David A. Gaul, ${ }^{\dagger}$ Nael A. McCarty, ${ }^{\S}$ Arlene Stecenko, ${ }^{*} \S$ \\ and Facundo M. Fernández ${ }^{*} \dagger$ (1) \\ ${ }^{\dagger}$ School of Chemistry and Biochemistry, Georgia Institute of Technology, Atlanta, Georgia 30332, United States \\ ${ }^{\ddagger}$ Centro de Investigaciones en Bionanociencias (CIBION), Consejo Nacional de Investigaciones Científicas y Técnicas \\ (CONICET), Godoy Cruz 2390, Ciudad de Buenos Aires C1425FQD, Argentina \\ ${ }^{\S}$ Emory + Children's Center for Cystic Fibrosis and Airways Disease Research and Department of Pediatrics, Emory University \\ School of Medicine and Children's Healthcare of Atlanta, Atlanta, Georgia 30322, United States
}

Supporting Information

ABSTRACT: The most common cause of death in cystic fibrosis (CF) patients is progressive lung function decline, which is punctuated by acute pulmonary exacerbations (APEs). A major challenge is to discover biomarkers for detecting an oncoming APE and allow for pre-emptive clinical interventions. Metabolic profiling of exhaled breath condensate (EBC) samples collected from CF patients before, during, and after APEs and under stable conditions $(n=210)$ was performed using ultraperformance liquid chromatography (UPLC) coupled to Orbitrap mass spectrometry (MS). Negative ion mode MS data showed that classification between metabolic profiles from "pre-APE" (pending APE before the CF patient had any signs of illness) and stable CF samples was

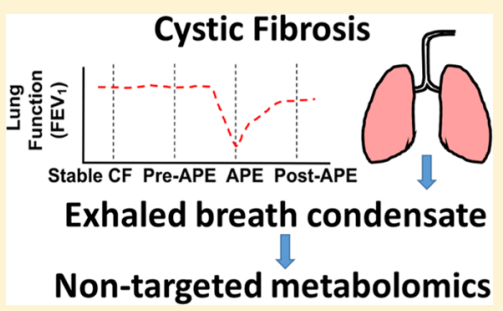
possible with good sensitivities ( 85.7 and $89.5 \%$ ), specificities (88.4 and $84.1 \%$ ), and accuracies (87.7 and $85.7 \%)$ for pediatric and adult patients, respectively. Improved classification performance was achieved by combining positive with negative ion mode data. Discriminant metabolites included two potential biomarkers identified in a previous pilot study: lactic acid and 4hydroxycyclohexylcarboxylic acid. Some of the discriminant metabolites had microbial origins, indicating a possible role of bacterial metabolism in APE progression. The results show promise for detecting an oncoming APE using EBC metabolites, thus permitting early intervention to abort such an event.

KEYWORDS: mass spectrometry, metabolomics, cystic fibrosis, exacerbation

\section{INTRODUCTION}

In cystic fibrosis (CF), the frequency and severity of acute pulmonary exacerbations (APEs) are important determinants for accelerated lung function decline and increased mortality. ${ }^{1,2}$ The CF Foundation Patient Registry shows that 24\% of children with CF and $43 \%$ of adults with CF had one or more APEs that required intravenous antibiotics treatment in $2017 .^{3}$ There are no consensus diagnostic criteria for CF APEs, as most of the current criteria are based on empirical data that has not been formally validated and may thus cause problematic treatment decisions. ${ }^{4}$ In addition, there is no pre-emptive screening method for stable CF patients to signal an oncoming APE, which hinders the implementation of an early intervention. ${ }^{5}$ These drawbacks emphasize the need for better biomarkers to improve APE diagnosis and to predict oncoming APE events.

Biomarkers indicating on-going CF APEs have been proposed for various types of biofluids. Only a few, however, have been demonstrated to be predictive of exacerbation. The most extensively studied blood biomarker is the C-reactive protein, which was found to correlate with CF disease severity with a significant increase in APEs compared to baseline in stable CF patients and a significant decrease after treatment. ${ }^{6}$
Compared to proteins, metabolites are closer in proximity to the phenotype and their changes can better reflect the status of a biological system. ${ }^{7}$ Several metabolomics studies have revealed detectable metabolic alterations associated with $\mathrm{CF}$ APEs. In a plasma metabolomics study by Laguna et al., for example, metabolic profiles of matched paired samples from 25 CF patients collected during an APE and during outpatient clinic visits were analyzed by liquid chromatography (LC) mass spectrometry (MS) and gas chromatography-MS. ${ }^{8}$ Five out of 398 identified metabolites showed significant alterations between stable CF patients and patients undergoing an APE, including hypoxanthine, N4-acetylcytidine, N-acetylmethionine, mannose, and cortisol. ${ }^{8}$ Quinn et al. identified the platelet activating factor and related lipids as potential biomarkers for CF APEs in sputum from $11 \mathrm{CF}$ patients, with the advantage that this biofluid is less invasive to collect than blood. ${ }^{9}$ However, most young children and many $\mathrm{CF}$ teens do not expectorate sputum when clinically stable. Thus, the use of sputum targets those adult patients with established airway disease and holds less promise for detecting disease in

Received: July 3, 2019

Published: October 17, 2019 
pediatric patients. Exhaled breath condensate $(\mathrm{EBC})$ is another biofluid that can be analyzed to establish differences prior to and during an APE, with the advantage that it can be easily collected from patients of all ages, including small children and with a wide variety of airway disease severity, including those with mild disease and little sputum production. ${ }^{10}$ Despite this potential usefulness, only one study ${ }^{11}$ has investigated the discrimination of stable CF patients $(n=29)$ from CF patients during exacerbations $(n=24)$. By using nuclear magnetic resonance (NMR) spectroscopy, ethanol, acetic acid, 2propanol, and methanol were identified as discriminating EBC metabolites. ${ }^{11}$ Together with our prior pilot study of 35 CF subjects where we identified metabolites present in EBC several weeks before an APE became clinically apparent, ${ }^{12}$ these investigations suggest that EBC metabolomics has the potential of revealing the biological pathways altered in the development and progression of an APE in CF, prompting the examination of a larger and better controlled patient cohort.

We here present an EBC metabolomics study of a patient cohort that included 97 stable CF patients, 36 pre-APE patients, $41 \mathrm{APE}$ patients and 36 post-APE patients, with two aims: (a) to compare the results with those from our pilot study and (b) to identify more robust EBC biomarkers for CF $\mathrm{APE}$ prediction and detection in adult and pediatric patients.

\section{MATERIALS AND METHODS}

Details on chemical reagents, EBC sample collection, and preparation for analysis, instrumental settings for metabolic profiling, metabolite identification by ion mobility (IM), and detailed data analysis procedures are provided in the Supporting Information section. All datasets are shared in the Metabolomics Workbench (https://www. metabolomicsworkbench.org/) with doi: 10.21228/M8409J.

\section{Cohort Description}

CF patients were seen in the clinic every three months when stable and more frequently with exacerbations. EBC was collected during these regular clinic visits to the Emory + Children's CF Care Center in Atlanta, Georgia, after obtaining informed consent. The patient's clinical course was then followed over the subsequent months so that they could be grouped according to their APE status. Clinically stable CF was defined as CF subjects whose symptoms were at the baseline, physical examination of the lungs was at the baseline, percent predicted forced expiratory volume in $1 \mathrm{~s}\left(\mathrm{ppFEV}_{1}\right)$ was within $10 \%$ of the yearly baseline, and no new therapies (particularly antibiotics) were added at that clinic visit; the patient was seen at the next clinic visit three months later and was again classified as clinically stable. EBC that was collected at the first of these paired clinically stable clinic visits was used for metabolic profiling of the clinically stable patient cohort as there was no APE identified in the three months following EBC collection. Ninety-seven CF subjects (age range 5-66, $50 \%$ female) met this definition of clinically stable without an APE in the subsequent 3 months. A severe APE was defined as an increase in respiratory symptoms (cough and sputum production) and/or changes in physical examination of the lungs (increase in crackles and decrease in airflow), at least a $10 \%$ decrease in baseline $\mathrm{ppFEV}_{1}$, and (in the opinion of the clinician) requiring hospitalization for treatment of the APE. In many instances, this was because outpatient treatment failed to restore the patient to baseline. Thus, use of the criteria that $\mathrm{ppFEV}_{1}$ was decreased by at least $10 \%$ of baseline and that hospitalization was required resulted in the study of more severe APEs that did not respond to outpatient treatment. EBC was collected in 41 of such subjects with a severe APE at the time of hospitalization (age range $8-58,56 \%$ female). In 36 subjects, EBC was collected 1-3 months after an APE event requiring hospitalization, labeled as post-APE (age range $8-64,64 \%$ female). Finally, EBC was collected in 36 subjects who were clinically stable as defined above but in the subsequent 1-3 months developed APEs severe enough to require hospitalization, labeled as pre-APE (age range 8-61, $61 \%$ female). Because of the wide age distribution in the patient cohort, samples were separated into adult (age >18) and pediatric (age $\leq 18)$ groups within each class. Attempts to classify samples by type of bacterial infection (e.g., Pseudomonas vs MSSA vs MRSA) or CF-related diabetes status did not produce any obvious subgroups and were not further pursued. Sample data from patients of pediatric and adult groups were preprocessed and filtered, and these features were analyzed by principal component analysis (PCA); outliers outside $95 \%$ confidence intervals were removed. Subsequently, orthogonal partial least-squares-discriminant-analysis (oPLSDA) binary classification models were performed for age- and gender-matched samples in each group (Table S1). For stable $\mathrm{CF}$ versus pre-APE comparisons, there were 3 pediatric and 9 adult patients that provided both a stable CF and a pre-APE sample. For the stable CF versus APE comparison, there were 4 pediatric and 2 adult patients providing both a stable CF and an APE sample.

\section{Metabolic Profiling by Ultraperformance Liquid} Chromatography-MS

Ultraperformance liquid chromatography (UPLC)-MS metabolic profiling analyses were performed on a Dionex Ultimate 3000 system (Thermo Scientific, Dionex, Sunnyvale, California, USA) equipped with a Waters ACQUITY UPLC BEH $\mathrm{C}_{18}$ column $(2.1 \times 50 \mathrm{~mm}, 1.7 \mu \mathrm{m}$ particle size, Waters Corporation, Milford, MA, USA), coupled to a Thermo Scientific Q Exactive HF hybrid quadruple-Orbitrap mass spectrometer. The main mass spectrometer parameters were as follows: capillary voltage $3.1 \mathrm{kV}$, capillary temperature $300{ }^{\circ} \mathrm{C}$, S-lens RF 50.0\%, sheath gas, auxiliary gas and sweep gas flow rate 48,11 , and 2 arbitrary units, respectively, and auxiliary gas heater temperature $413{ }^{\circ} \mathrm{C}$. The $\mathrm{m} / z$ scan range was $50.0-$ 750.0 with an automatic gain control (AGC) target of $3 \times 10^{6}$ and maximum injection time of $512 \mathrm{~ms}$. The mass resolution setting was 240000 . For UPLC-MS/MS experiments, the product ion-mass spectra were acquired with a resolution of 30000 , AGC target of $1 \times 10^{5}$, maximum injection time of 64 ms, isolation window of $0.4 \mathrm{~m} / z$ and normalized collision cell voltages between 10 and $100 \mathrm{~V}$, depending on the analyte. Additional details are provided in the Supporting Information section.

\section{Metabolite Identification and Pathway Analysis}

Spectral features with tentative candidates in the Human Metabolome Database (HMDB) ${ }^{13}$ were targeted for further identification based on (i) their accurate mass and isotopic pattern, (ii) tandem MS experiments, where the respective precursor ions were quadrupole-selected and -activated, and search of MS/MS spectra in the Metlin database, ${ }^{14}$ and (iii) further validation against chemical standards (when available). For those cases in which MS/MS spectra were not available in the Metlin database for comparison, fragmentation patterns were manually interpreted for metabolite annotation. Com- 


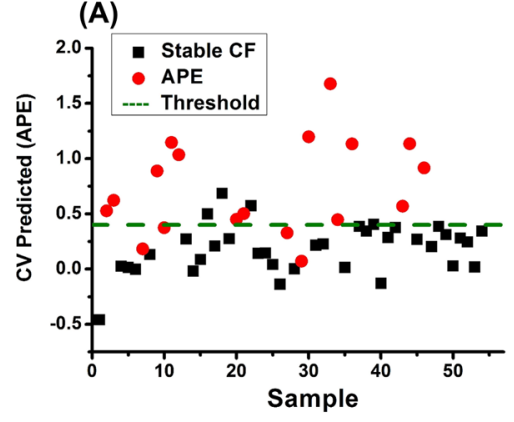

(C)

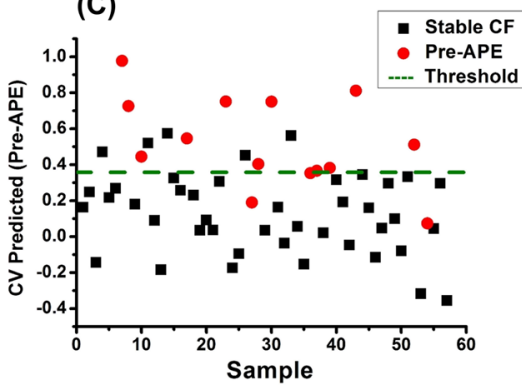

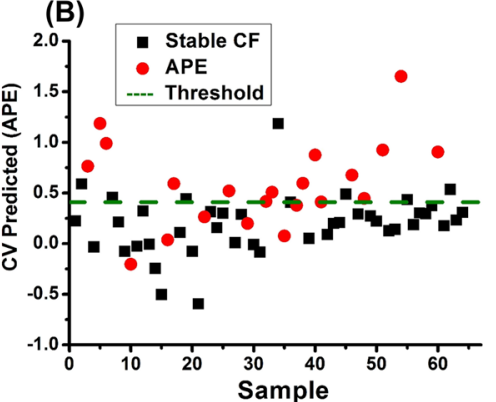

(D)

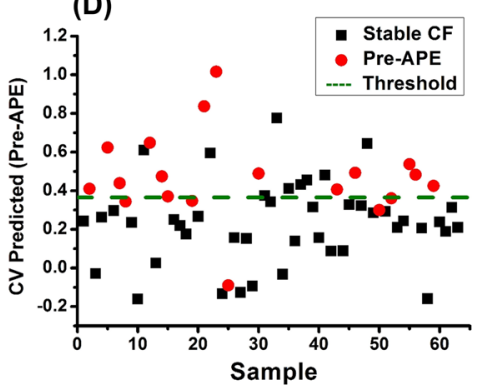

Figure 1. oPLS-DA cross-validated classification plots using negative ion mode data, including comparison of APE vs stable CF samples in age- and gender-matched EBC samples from pediatric patients (A) and those from adult patients (B), and comparison of pre-APE vs stable CF samples in age- and gender-matched EBC samples from pediatric patients (C) and those from adult patients (D). The $x$-axis represents the sample number, and $y$-axis represents the cross-validated predicted scores of the oPLS-DA classification model. APE/pre-APE and stable CF samples are represented by red circles and black squares, respectively. The decision threshold is represented as a green dashed line.

mercially available standards were analyzed under identical conditions as EBC samples to validate putative metabolite identities by chromatographic retention time $\left(R_{t}\right)$ matching and MS/MS fragmentation pattern matching. For metabolites with $R_{t}$ at the solvent front, collision cross-section (CCS) values were measured using IM MS to provide an additional molecular descriptor to increase identification confidence by comparing to those of chemical standards (see the Supporting Information section). Pathway analysis was performed with Metaboanalyst v3.0. ${ }^{15}$ A total of 23 uniquely identified discriminant metabolites from all panels (metabolites in Tables S3 and S4) were chosen for analysis. Data were autoscaled, and other parameters were kept with default settings.

\section{RESULTS AND DISCUSSION}

\section{Data Extraction Results}

A total of 1334 and 923 features were obtained from UPLCMS data in negative and positive ion modes, respectively. Adducts, in-source fragment ions, and chloride salt cluster ions were grouped according to criteria in Table S2. The quality control sample-based robust locally estimated scatterplot smoothing (LOESS) signal correction (QC-RLSC) was applied to the data, followed by removal of features with relative standard deviation $>30 \%$ in quality control (QC) samples. ${ }^{16}$ After blank correction, features with zero values in $>80 \%$ of samples considering all classes (stable CF, pre-APE, $\mathrm{APE}$, and post-APE) were removed, leaving 1015 and 635 features in negative and positive ion mode data, respectively. Subsequently, contaminants and electrospray ionization (ESI) artifacts such as chloride salt cluster ions and high mass defect ions defined by the McMillan filter ${ }^{17}$ were removed, leaving 745 and 538 features in negative and positive ion mode data, respectively. The resulting data matrices were normalized by total ion intensity and then passed through a $50 \%$ sample prevalence filter that preserved features present in $\geq 50 \%$ samples within one of the sample groups used for binary classification, yielding 281 and 262 features in negative and positive ion mode data for APE/stable CF classes, and 278 and 268 features in negative and positive ion mode data for pre$\mathrm{APE} /$ stable CF classes. The remaining features were then searched in the HMDB and only those with endogenous human or microbial candidate identities were retained. This putative identity filter was performed to ensure the features used for multivariate analysis had a high chance of being identified as human endogenous or microbial compounds and minimize false positive identifications. One limitation of this approach, however, is that it sacrifices the chances of discovering potentially novel discriminant compounds. Following this step, 58 and 46 features remained in negative and positive ion mode data for the APE/stable CF class pair, and 57 and 44 compounds for the pre-APE/stable CF class pair. These remaining features constituted the final datasets used for subsequent multivariate analyses. EBC metabolome fingerprints are illustrated in heat maps (Figure S1) for visualization of differences among features across all sample classes from pediatric and adult patients. In the pediatric group, APE metabolic profiles showed differences from the other three groups, with more compounds increasing in relative abundance; in the adult group, metabolic profiles for APE EBC samples were similar to those of post-APE samples. For both pediatric and adult groups, the distinction between pre$\mathrm{APE}$ and stable CF metabolic profiles was not obvious in the heat maps. PCA was performed for each age group with the final set of features after data preprocessing and filtering steps, and outliers outside $95 \%$ confidence intervals were removed. For combined negative and positive mode data, there were 1 adult pre-APE outlier, and 1 pediatric and 4 adult stable CF outliers for the pre-APE versus stable CF comparison, and 3 pediatric and 3 adult APE outliers and 4 adult stable CF 
Table 1. Identification of Discriminant Features in oPLS-DA Classification of APE Samples from Stable CF Samples Using Negative Ion Mode Data

\begin{tabular}{|c|c|c|c|c|c|c|c|}
\hline $\begin{array}{l}\text { age-based } \\
\text { patient } \\
\text { group }\end{array}$ & $m / z$ & $\underset{(\mathrm{min})}{R_{t}}$ & ion type & tentative identity & $\begin{array}{l}\text { elemental } \\
\text { formula }\end{array}$ & $\begin{array}{c}\text { median fold } \\
\text { change } \\
\text { (APE/stable CF) }\end{array}$ & identification method \\
\hline \multirow[t]{9}{*}{ pediatric } & 89.0232 & 0.55 & {$[\mathrm{M}-\mathrm{H}]^{-}$} & lactic acid & $\mathrm{C}_{3} \mathrm{H}_{6} \mathrm{O}_{3}$ & 2.5 & $\mathrm{CCS}^{a}$ and MS/MS ${ }^{a, b}$ \\
\hline & 128.0342 & 0.53 & {$[\mathrm{M}-\mathrm{H}]^{-}$} & pyroglutamic acid & $\mathrm{C}_{5} \mathrm{H}_{7} \mathrm{NO}_{3}$ & 1.5 & $\mathrm{CCS}^{a}$ and $\mathrm{MS} / \mathrm{MS}^{a, b}$ \\
\hline & 127.0503 & 0.87 & {$[\mathrm{M}-\mathrm{H}]^{-}$} & dihydrothymine & $\mathrm{C}_{5} \mathrm{H}_{8} \mathrm{~N}_{2} \mathrm{O}_{2}$ & 3.0 & $\mathrm{MS} / \mathrm{MS}^{c}$ \\
\hline & 59.0125 & 0.56 & {$[\mathrm{M}-\mathrm{H}]^{-}$} & acetic acid & $\mathrm{C}_{2} \mathrm{H}_{4} \mathrm{O}_{2}$ & -1.7 & $\mathrm{CCS}^{a}$ \\
\hline & 130.0499 & 0.57 & {$[\mathrm{M}-\mathrm{H}]^{-}$} & & $\mathrm{C}_{5} \mathrm{H}_{9} \mathrm{NO}_{3}$ & 1.6 & $d$ \\
\hline & 143.0340 & 0.58 & {$[\mathrm{M}-\mathrm{H}]^{-}$} & $\begin{array}{l}\text { 3-methylglutaconic acid, } \\
\text { 2-methylglutaconic acid, 2-hexenedioic } \\
\text { acid, 3-hexenedioic acid }\end{array}$ & $\mathrm{C}_{6} \mathrm{H}_{8} \mathrm{O}_{4}$ & -1.4 & $\begin{array}{l}\mathrm{MS} / \mathrm{MS}^{c} \text { for } 2 \text {-hexenedioic } \\
\text { acid, MS/MS }{ }^{a} \text { for the other } \\
\text { candidates }\end{array}$ \\
\hline & 227.1036 & 0.66 & {$[\mathrm{M}-\mathrm{H}]^{-}$} & prolylhydroxyproline & $\mathrm{C}_{10} \mathrm{H}_{16} \mathrm{~N}_{2} \mathrm{O}_{4}$ & 1.8 & $\mathrm{MS} / \mathrm{MS}^{c}$ \\
\hline & 165.0549 & 1.77 & {$[\mathrm{M}-\mathrm{H}]^{-}$} & & $\mathrm{C}_{9} \mathrm{H}_{10} \mathrm{O}_{3}$ & 2.4 & d \\
\hline & 187.0064 & 0.64 & {$[\mathrm{M}-\mathrm{H}]^{-}$} & & $\mathrm{C}_{7} \mathrm{H}_{8} \mathrm{O}_{4} \mathrm{~S}$ & $\mathrm{~N} / \mathrm{A}^{e}$ & $d$ \\
\hline \multirow[t]{10}{*}{ adult } & 143.0704 & 0.67 & {$[\mathrm{M}-\mathrm{H}]^{-}$} & 4-hydroxycyclohexylcarboxylic acid & $\mathrm{C}_{7} \mathrm{H}_{12} \mathrm{O}_{3}$ & 1.5 & $\mathrm{CCS}^{a}$ and $\mathrm{MS} / \mathrm{MS}^{a, c}$ \\
\hline & 187.0970 & 0.63 & {$[\mathrm{M}-\mathrm{H}]^{-}$} & nonanedioic acid, azelaic acid & $\mathrm{C}_{9} \mathrm{H}_{16} \mathrm{O}_{4}$ & 2.5 & $\mathrm{CCS}^{a}$ and $\mathrm{MS} / \mathrm{MS}^{a, b}$ \\
\hline & 201.1128 & 1.07 & {$[\mathrm{M}-\mathrm{H}]^{-}$} & sebacic acid & $\mathrm{C}_{10} \mathrm{H}_{18} \mathrm{O}_{4}$ & 1.6 & $R_{t}^{a}$ and $\mathrm{MS} / \mathrm{MS}^{a, b}$ \\
\hline & 85.0283 & 0.61 & {$[\mathrm{M}-\mathrm{H}]^{-}$} & $\gamma$-butyrolactone, oxolan-3-one & $\mathrm{C}_{4} \mathrm{H}_{6} \mathrm{O}_{2}$ & 1.6 & $\mathrm{MS} / \mathrm{MS}^{c}$ \\
\hline & 115.0389 & 0.59 & {$[\mathrm{M}-\mathrm{H}]^{-}$} & levulinic acid & $\mathrm{C}_{5} \mathrm{H}_{8} \mathrm{O}_{3}$ & $\mathrm{~N} / \mathrm{A}$ & $\mathrm{MS} / \mathrm{MS}^{a, c}$ \\
\hline & 157.0862 & 0.97 & {$[\mathrm{M}-\mathrm{H}]^{-}$} & 4-hydroxycyclohexylacetic acid & $\mathrm{C}_{8} \mathrm{H}_{14} \mathrm{O}_{3}$ & 1.5 & $\mathrm{MS} / \mathrm{MS}^{c}$ \\
\hline & 59.0125 & 0.56 & {$[\mathrm{M}-\mathrm{H}]^{-}$} & acetic acid & $\mathrm{C}_{2} \mathrm{H}_{4} \mathrm{O}_{2}$ & -1.4 & $\mathrm{CCS}^{a}$ \\
\hline & 165.0549 & 1.77 & {$[\mathrm{M}-\mathrm{H}]^{-}$} & & $\mathrm{C}_{9} \mathrm{H}_{10} \mathrm{O}_{3}$ & 3.3 & $d$ \\
\hline & 168.0658 & 0.73 & {$[\mathrm{M}-\mathrm{H}]^{-}$} & & $\mathrm{C}_{8} \mathrm{H}_{11} \mathrm{NO}_{3}$ & -1.3 & $d$ \\
\hline & 177.0397 & 0.53 & {$[\mathrm{M}-\mathrm{H}]^{-}$} & & $\mathrm{C}_{6} \mathrm{H}_{10} \mathrm{O}_{6}$ & 1.6 & $d$ \\
\hline
\end{tabular}

${ }^{a}$ Match to chemical standard. ${ }^{b}$ Match to Metlin MS/MS. ${ }^{c}$ Match to manual analysis. ${ }^{d}$ No endogenous ID, not match to chemical standard, match to Metlin MS/MS or match to manual analysis. ${ }^{e}$ Unable to calculate median fold change because the denominator is 0 .

Table 2. Identification of Discriminant Features in oPLS-DA Classification of Pre-APE Samples from Stable CF Samples Using Negative Ion Mode Data

\begin{tabular}{|c|c|c|c|c|c|c|c|}
\hline $\begin{array}{l}\text { age-based } \\
\text { patient group }\end{array}$ & $m / z$ & $\begin{array}{c}R_{t} \\
(\mathrm{~min})\end{array}$ & ion type & tentative identity & $\begin{array}{l}\text { elemental } \\
\text { formula }\end{array}$ & $\begin{array}{l}\text { median fold change } \\
\text { (pre-APE/stable CF) }\end{array}$ & identification method \\
\hline \multirow[t]{11}{*}{ pediatric } & 89.0232 & 0.55 & {$[\mathrm{M}-\mathrm{H}]^{-}$} & lactic acid & $\mathrm{C}_{3} \mathrm{H}_{6} \mathrm{O}_{3}$ & 1.3 & $\mathrm{CCS}^{a}$ and $\mathrm{MS} / \mathrm{MS}^{a, b}$ \\
\hline & 133.0132 & 0.86 & {$[\mathrm{M}-\mathrm{H}]^{-}$} & malic acid & $\mathrm{C}_{4} \mathrm{H}_{6} \mathrm{O}_{5}$ & $-2.0 \times 10^{2}$ & $\mathrm{Rt}^{a}$ and $\mathrm{MS} / \mathrm{MS}^{a, b}$ \\
\hline & 189.0762 & 0.59 & {$[\mathrm{M}-\mathrm{H}]^{-}$} & 3-hydroxysuberic acid & $\mathrm{C}_{8} \mathrm{H}_{14} \mathrm{O}_{5}$ & 1.4 & $\mathrm{MS} / \mathrm{MS}^{c}$ \\
\hline & 329.2338 & 6.73 & {$[\mathrm{M}-\mathrm{H}]^{-}$} & 9,10,13-TriHOME & $\mathrm{C}_{18} \mathrm{H}_{34} \mathrm{O}_{5}$ & 1.3 & $\mathrm{MS} / \mathrm{MS}^{c}$ \\
\hline & 73.0277 & 0.64 & {$[\mathrm{M}-\mathrm{H}]^{-}$} & propionic acid & $\mathrm{C}_{3} \mathrm{H}_{6} \mathrm{O}_{2}$ & -1.1 & $\mathrm{CCS}^{a}$ and $\mathrm{MS} / \mathrm{MS}^{a, c}$ \\
\hline & 130.0499 & 0.57 & {$[\mathrm{M}-\mathrm{H}]^{-}$} & & $\mathrm{C}_{5} \mathrm{H}_{9} \mathrm{NO}_{3}$ & 1.9 & $d$ \\
\hline & 145.0973 & 0.70 & {$[\mathrm{M}-\mathrm{H}]^{-}$} & lysine & $\mathrm{C}_{6} \mathrm{H}_{14} \mathrm{~N}_{2} \mathrm{O}_{2}$ & 2.9 & $\begin{array}{l}R_{t}^{a} \text { and } \mathrm{MS} / \mathrm{MS}^{a, b} \text { for } \\
\text { positive mode }\end{array}$ \\
\hline & 101.0596 & 0.80 & {$[\mathrm{M}-\mathrm{H}]^{-}$} & & $\mathrm{C}_{5} \mathrm{H}_{10} \mathrm{O}_{2}$ & $\mathrm{~N} / \mathrm{A}^{e}$ & $d$ \\
\hline & 122.0237 & 1.40 & {$[\mathrm{M}-\mathrm{H}]^{-}$} & & $\mathrm{C}_{6} \mathrm{H}_{5} \mathrm{NO}_{2}$ & $\mathrm{~N} / \mathrm{A}$ & $d$ \\
\hline & 187.0064 & 0.64 & {$[\mathrm{M}-\mathrm{H}]^{-}$} & & $\mathrm{C}_{7} \mathrm{H}_{8} \mathrm{O}_{4} \mathrm{~S}$ & $\mathrm{~N} / \mathrm{A}$ & $d$ \\
\hline & 213.1493 & 5.97 & {$[\mathrm{M}-\mathrm{H}]^{-}$} & & $\mathrm{C}_{12} \mathrm{H}_{22} \mathrm{O}_{3}$ & 1.6 & $d$ \\
\hline \multirow[t]{5}{*}{ adult } & 143.0704 & 0.67 & {$[\mathrm{M}-\mathrm{H}]^{-}$} & 4-hydroxycyclohexylcarboxylic acid & $\mathrm{C}_{7} \mathrm{H}_{12} \mathrm{O}_{3}$ & 1.8 & $\mathrm{CCS}^{a}$ and $\mathrm{MS} / \mathrm{MS}^{a, c}$ \\
\hline & 137.0234 & 0.74 & {$[\mathrm{M}-\mathrm{H}]^{-}$} & salicylic acid & $\mathrm{C}_{7} \mathrm{H}_{6} \mathrm{O}_{3}$ & 2.5 & $R_{t}^{a}$ and $\mathrm{MS} / \mathrm{MS}^{a, b}$ \\
\hline & 157.0862 & 0.97 & {$[\mathrm{M}-\mathrm{H}]^{-}$} & 4-hydroxycyclohexylacetic acid & $\mathrm{C}_{8} \mathrm{H}_{14} \mathrm{O}_{3}$ & 2.1 & $\mathrm{MS} / \mathrm{MS}^{c}$ \\
\hline & 171.0655 & 0.64 & {$[\mathrm{M}-\mathrm{H}]^{-}$} & $\begin{array}{l}\text { 2-octenedioic acid, 4-octenedioic } \\
\text { acid, 3-octenedioic acid }\end{array}$ & $\mathrm{C}_{8} \mathrm{H}_{12} \mathrm{O}_{4}$ & 2.0 & $\mathrm{MS} / \mathrm{MS}^{c}$ \\
\hline & 130.0499 & 0.57 & {$[\mathrm{M}-\mathrm{H}]^{-}$} & & $\mathrm{C}_{5} \mathrm{H}_{9} \mathrm{NO}_{3}$ & 1.0 & $d$ \\
\hline
\end{tabular}

${ }^{a}$ Match to chemical standard. ${ }^{b}$ Match to Metlin MS/MS. ${ }^{c}$ Match to manual analysis. ${ }^{d}$ No endogenous ID, not match to chemical standard, match to Metlin MS/MS or match to manual analysis. Significant median fold change is shown in italics. ${ }^{e}$ Unable to calculate median fold change because the denominator is 0 .

outliers for the APE versus stable CF comparison. Then, interval PLS-DA (iPLS-DA) feature selection and oPLS-DA binary classification models were built for age- and gendermatched samples in each age-based group (Table S1).

\section{Classification Performance}

Data analysis of negative ion mode data showed that discrimination of APE from stable CF samples was possible in both adult and pediatric groups (Figure 1 and Table S3).
For classification of pediatric patients, nine discriminant features were selected by iPLS-DA, yielding a sensitivity of $83.3 \%$, specificity of $91.7 \%$, and accuracy of $88.9 \%$. For the adult group, the oPLS-DA model provided a sensitivity of $76.2 \%$, specificity of $83.7 \%$, and accuracy of $81.3 \%$ in distinguishing 21 APE samples from 43 stable CF samples, using a panel of 10 discriminant features with six latent variables. For discrimination of pre-APE samples from stable 
CF EBC samples, another set of oPLS-DA models was built that provided sensitivities of 85.7 and $89.5 \%$, specificities of 88.4 and $84.1 \%$, and accuracies of 87.7 and $85.7 \%$ for differentiating samples of 14 pre-APE pediatric patients or 19 adult patients from 43 stable pediatric patients or 44 adult patients, respectively. All models had good area under the receiver operating characteristic curve (AUC) values ranging from 0.8 to 0.9 , with permutation testing showing no overfitting.

Data analysis was also performed on the dataset combining positive and negative ion mode spectral features. Results showed classification was also feasible when samples were separated into adult and pediatric groups and improved classification performance was obtained for most comparisons with accuracies enhanced between 3 and 9.3\% (Table S4 and Figure S2). All models had high AUC values of approximately 0.9 , and no indication of overfitting as suggested by permutation test results.

It is worth mentioning that attempts were initially performed to discriminate between pre-APE and stable CF patients without splitting the cohort into adult and pediatric subgroups, but results were not satisfactory. For example, using the combined negative and positive mode LC-MS data, the sensitivity and specificity obtained from the oPLS-DA classification model for the pre-APE versus stable CF binary comparison were lower ( 74.3 and $88.0 \%$, respectively, Figure S3) than those obtained when separated pediatric (100 and $87.5 \%)$ and adult (85.7 and 97.7\%) patient cohorts were analyzed (Table S4). In addition, when lactic acid abundance was plotted against patient age for pre-APE and stable CF samples, different levels were observed for pediatric and adult patients (Figure S4). Overall, the average lactic acid level in pediatric patients was significantly higher than that in adult patients $\left(p=1.3 \times 10^{-5}\right)$. Based on these results, samples were grouped into adult and pediatric groups for classification with a cut-off age of 18 years of age.

\section{Identification of Discriminant Metabolites and Their Biological Relevance}

Experimental MS/MS spectra of discriminant features were matched to entries in the Metlin database or manually analyzed if no database spectra were available. In addition, if a chemical standard was available, both retention time $\left(R_{t}\right)$ and MS/MS spectrum of the discriminant feature were compared to it. Because some features co-eluted with the chromatographic solvent front $\left(R_{t}=0.5-0.7 \mathrm{~min}\right)$, CCS was used as an additional molecular descriptor for identification instead of $R_{t}$. A more confident identity is reported if CCS values matched with chemical standards within $2 \%$ (Tables 1 and 2 and Tables S5 and S6). Figure S5 shows an example of a positive-ion mode identification of a discriminant feature with $\mathrm{m} / \mathrm{z}$ 175.1188 by comparison of its MS/MS spectrum to the arginine chemical standard. In addition, $R_{t}$ and CCS were also matched (Table S6).

In this work, metabolic profiling of EBC samples was conducted with a larger patient cohort $(n=210)$ than our prior pilot study to investigate potential EBC biomarkers for APE detection and prediction by means of a nontargeted UPLC-MS-based method coupled to multivariate statistical analysis. Both negative ion mode data and combined positive and negative ion mode data showed classification of APE and pre-APE patients from stable CF patients in both adult and pediatric cohorts. Supervised multivariate statistical models yielded good classification accuracies ranging between 81.3 and 93.9\%, depending on the binary comparison, with AUC values of $\sim 0.8-0.9$, and provided different discriminant EBC metabolite panels for APE and pre-APE detection in adult and pediatric patients.

Of all discriminant features, some have been reported as being connected with microbial metabolism, re-emphasizing the important role that the CF microbiome has in terms of interacting with the host and participating in inflammatory mechanisms during and before the onset of an APE. Several discriminant metabolites showed interesting trends in their abundance changes in EBC samples from patients at different stages preceding and following an APE event, in pediatric cohorts, including lactic acid, leucine/isoleucine, and butyric acid (Figure 2).
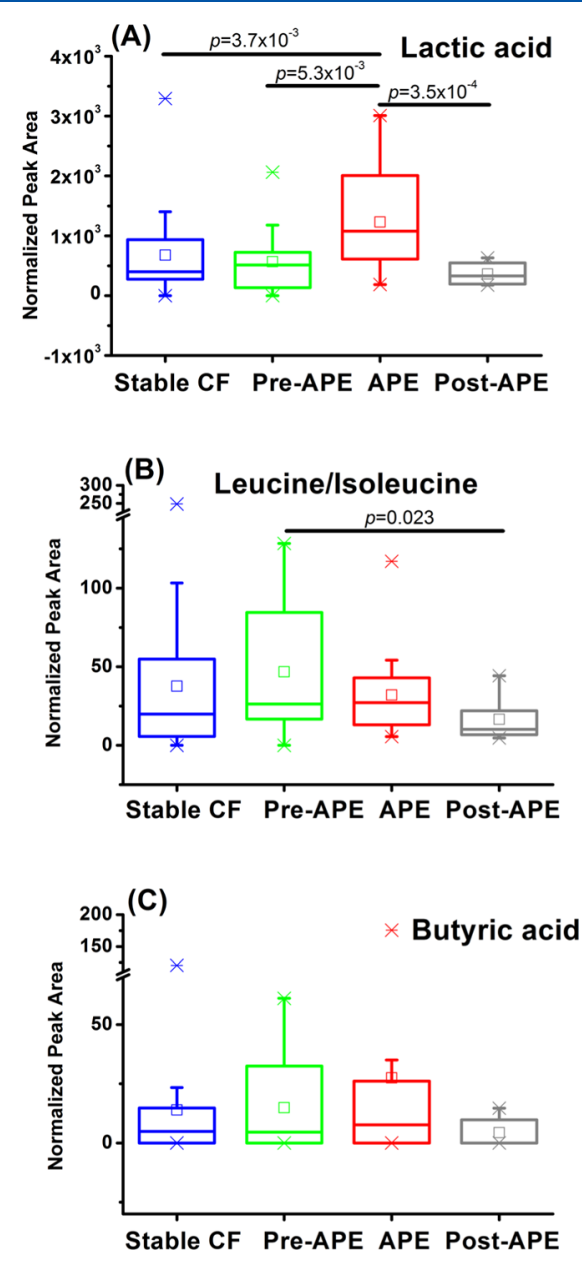

Figure 2. Box plots for discriminant metabolites with changing trends in pediatric patients: (A) lactic acid, (B) leucine and/or isoleucine, and $(C)$ butyric acid. Mean values are represented by an open square; median values are represented by a line in the box; the edges of the box are 25th and 75th percentiles; the whisker extends to the most extreme values in data not including outliers, with a $99.3 \%$ coverage; outliers are represented by asterisks.

In EBC samples from pediatric patients, lactic acid was significantly elevated in APE samples compared to stable, preand post-APE ones (Figure 2A). This compound was also selected by iPLS-DA in the model comparing EBC samples from pre-APE patients with those from stable CF patients in the pilot study. ${ }^{22}$ In the model comparing adult APE and stable 
(A)

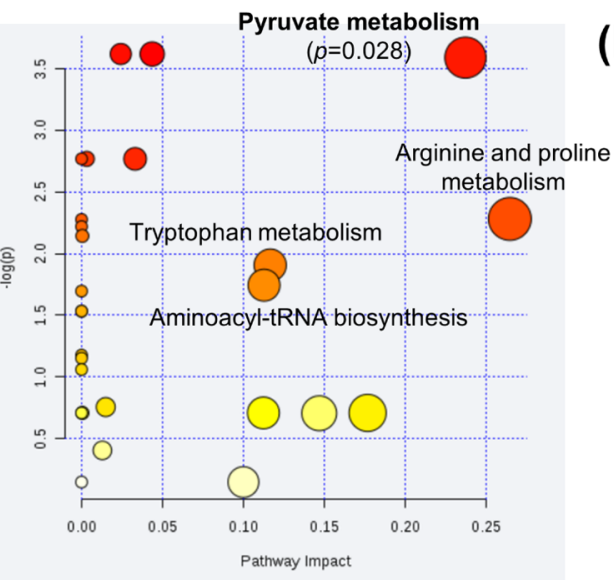

(C)

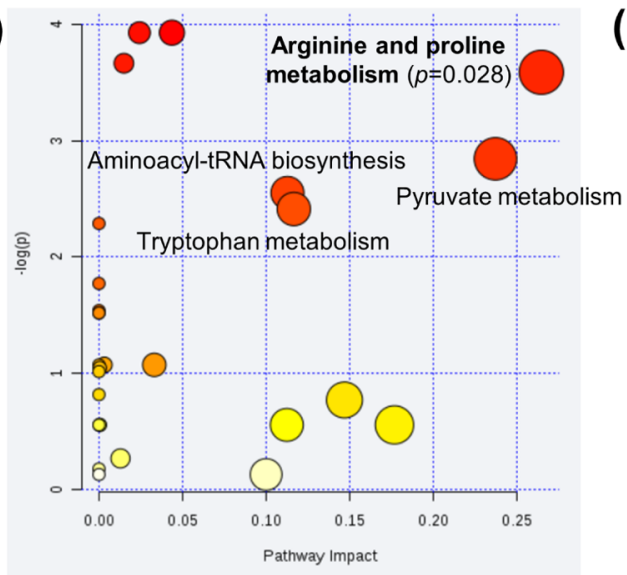

(B)

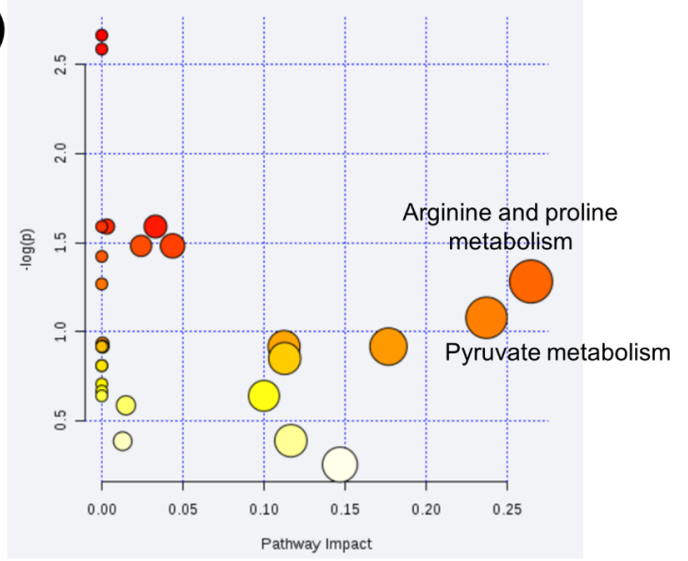

(D)

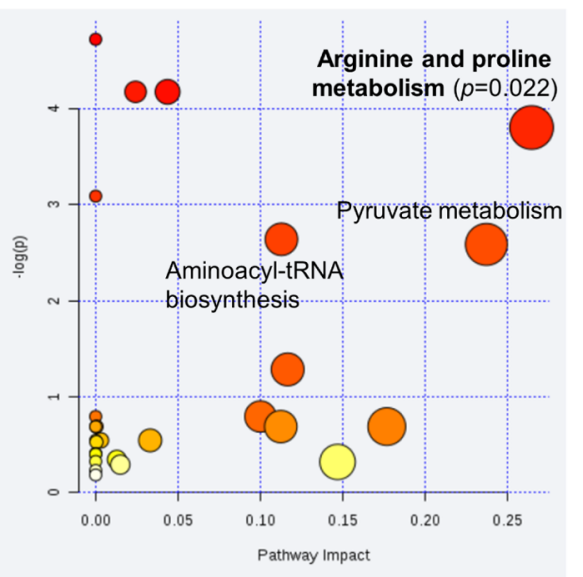

Figure 3. Pathway analysis of the 23 uniquely-identified discriminant metabolites from all panels, including EBC samples from APE vs stable CF (A) pediatric and (B) adult patients, and pre-APE vs stable CF in (C) pediatric and (D) adult patients. Each circle on the map represents a pathway, and the size and color of the circle are based on the $p$-value, indicating the significance of the changes in the matched metabolites in the pathway, and the pathway impact score, which is correlated with the centrality of the metabolites involved. ${ }^{15,38}$

CF samples, however, lactic acid was not included because compared to pediatric patients, adult patients had lower abundance of lactic acid in the APE group, and changes between the stable CF and APE groups were not statistically significant. Lactic acid is a fermentation metabolite of the anaerobic microbial community in $\mathrm{CF}$ and a potential biomarker associated with CF progression. ${ }^{18}$ In a sputum metabolomics study, lactic acid was detected with significantly increased levels in samples from APE patients compared to those from stable CF patients using LC-MS/MS. ${ }^{19}$ The mean abundance of leucine/isoleucine exhibited an increasing trend in EBC samples from stable CF to pre-APE pediatric patients, and a decreasing trend from pre-APE to APE, and from APE to post-APE (Figure $2 B$ ). The decrease in mean abundance from the pre-APE to the APE group in the cohort might be due to the fact that over $50 \%$ of the APE patients had already started intravenous antibiotic treatment at the time of EBC collection, resulting in a different inflammatory phenotype from that in pre-APE patients, who had not yet been aggressively treated. Leucine was reported as an important discriminant metabolite for classification of CF patients with high and low inflammation. ${ }^{20}$ It should also be noted that the catabolism of branched chain amino acids such as leucine and isoleucine is subjected to the carbon catabolite repression control in Pseudomonas aeruginosa, and the levels of other amino acids are also affected by this mechanism. ${ }^{21}$ Therefore, the levels of leucine or isoleucine together with other amino acids detected in this study may be confounded by the distribution of $P$. aeruginosa infection in patients. Butyric acid has been found to be secreted by anaerobic bacteria found in CF airways, and it was also detected in bronchoalveolar lavage fluid samples from CF patients. ${ }^{22}$ In this study, both the mean and median levels of butyric acid were higher in APE pediatric samples compared to stable CF and pre-APE ones (Figure 2C). Butyric acid, together with acetic acid and propionic acid, which also appeared in the discriminant feature panels (Tables 1 and 2 and S5 and S6), are short-chain fatty acids associated with immune and inflammatory processes in CF airways. ${ }^{22}$ Interestingly, acetic acid was identified as one of the potential EBC biomarkers for discriminating stable CF patients from unstable CF patients during exacerbations in a metabolomics study using NMR. ${ }^{11}$

Interestingly, 4-hydroxycyclohexanecarboxylic acid, which is a metabolite associated with gut microbial mammalian cometabolism and one of the two discriminant features in the model for APE versus stable CF sample classification in the pilot study, ${ }^{12}$ appeared in multiple discriminant panels in this larger cohort study (Tables 1 and 2 and S5 and S6). In addition, pyroglutamic acid, which is involved in the $\gamma$-glutamyl cycle and present in both APE versus stable CF and pre-APE versus stable CF discriminant panels in the pilot study, ${ }^{12}$ also appeared in the nine-feature negative mode data model 
differentiating APE from stable CF pediatric samples (Table 1). These metabolites, selected for discrimination in both studies in a nonsupervised fashion, corroborated the biological significance and implications for future diagnostic approaches of the panels described here.

Malic acid, which was present in the 11-feature negative ion mode oPLS-DA model for classification of pre-APE and stable CF pediatric patient samples, was significantly decreased in pre-APE, with a large median fold change of $2.0 \times 10^{2}$ (Table 2). Malic acid has been recently detected in exhaled breath for the first time by secondary ESI high-resolution $\mathrm{MS}^{23}$ and it was reported to have cardioprotective effects possibly due to its anti-inflammatory properties. ${ }^{24}$ In addition, it is involved in pyruvate metabolism, which was found to be significantly altered between APE and stable CF pediatric samples (Figure $3 \mathrm{~A})$. Free carnitine, which was identified in the adult APE versus stable CF discriminant panel of the model using combined negative and positive ion mode data had a 1.5 fold increase in APE samples (Table S5). Carnitine can be either synthesized in the human body or obtained from dietary red meat and metabolized by intestinal bacteria. ${ }^{25}$ It is involved in fatty acid transport to mitochondria, with abnormal fatty acid metabolism having been postulated to be associated with $\mathrm{CF}^{25-28}$ Interestingly, free carnitine levels in plasma have been reported to be significantly higher in CF patients compared to healthy controls. ${ }^{29}$ To the best of our knowledge, carnitine has not been previously detected in EBC. Formylanthranilic acid and oxindole (Tables S5 and S6) are involved in tryptophan metabolism, ${ }^{30}$ and tryptophan was also selected as a discriminant compound in the classification model of APE versus stable CF pediatric samples (Table S5). Tryptophan metabolism plays a crucial role in gut mucosal homeostasis and microbiome regulation, ${ }^{31}$ and it was found to be significantly altered in primary human airway epithelial cells from CF patients compared to those from non-CF individuals in a metabolic profiling study by Wetmore et al. ${ }^{32}$ Further investigation of the microbial metabolites and integration of CF microbiome studies with metabolomics should improve our current understanding of host-pathogen interactions in APE development and progression, guiding clinical decisions for early intervention and personalized APE treatment. ${ }^{33}$

Pathway analysis of the 23 uniquely identified discriminant metabolites from all panels revealed pathways with significant changes in APE and pre-APE compared to stable CF patients. These included arginine and proline metabolism, which were found to be significantly altered in pre-APE adult and pediatric samples, and pyruvate metabolism, which was significantly changed between APE and stable CF pediatric patients (Figure $3)$. Arginine was one of the 11 discriminant features responsible for classification of adult pre-APE from stable CF samples, with a 3.1-fold increase in the pre-APE group (Table S6). Its concentration in EBC has been shown to be associated with lung function, with a significant negative correlation with percent predicted forced vital capacity and a nearly significant negative correlation with percent predicted $\mathrm{FEV}_{1} \cdot{ }^{34}$ Proline and glutamic acid, identified as discriminant metabolites between pre-APE and stable CF pediatric patients, are downstream products of arginase. ${ }^{35}$ In this binary comparison, proline had a 2.4-fold decrease and glutamic acid had a 1.3-fold increase in abundance in pre-APE pediatric patients (Table S6). Significantly increased proline and glutamic acid levels were found after antibiotic treatment of $\mathrm{APE},{ }^{35}$ suggesting that further research is needed to elucidate the mechanisms involved in the observed alterations of this metabolic pathway during CF APE progression.

Pyruvate metabolism, including lactic acid and acetic acid, was found to be altered in EBC samples collected from pediatric patients during an APE compared to those from stable CF pediatric patients. Malic acid was also identified as being significantly altered in EBC samples collected from pediatric patients in the 3-month time window before an APE event. Pyruvate metabolism is at a key intersection of many pathways in human biological systems including glycolysis and the citric acid cycle, and abnormalities in pyruvate metabolism have been strongly associated with various diseases. ${ }^{36}$ The flux of pyruvate metabolism is highly diverse in different strains of $P$. aeruginosa, ${ }^{37}$ and its relationship to $\mathrm{CF}$ also warrants further investigation.

In conclusion, nontargeted EBC metabolic profiling combined with supervised multivariate classification models demonstrated good performance for detecting APEs and predicting an oncoming APE event. EBC metabolites exhibiting significant changes at different stages of an APE event were discussed in relation to the altered metabolic pathways and their microbial relevance. All three discriminant metabolites in the pilot study were also selected in this larger cohort in a nonsupervised fashion, validating the biological significance of the panels identified for APE detection and prediction. One limitation of the study, however, is the lack of sufficient number of longitudinal EBC samples collected from the same patient at different stages of APE progression for time series analysis. In addition, further investigation of the $\mathrm{CF}$ microbiome should aid to improve our current understanding of the link between host and pathogen interactions in APE development and progression and to obtain chemical information on pathogen-specific metabolites that may assist in personalized clinical decisions for early intervention and better APE treatment.

\section{ASSOCIATED CONTENT}

\section{Supporting Information}

The Supporting Information is available free of charge on the ACS Publications website at DOI: 10.1021/acs.jproteome.9b00443.

Materials and Methods, chemicals, EBC sample collection and preparation, metabolic profiling by UPLC-MS, flow injection-traveling wave IM-MS analysis, data analysis, age- and gender-matched samples in the studied patient cohort, criteria applied for grouping adduct ions, in-source fragment ions, and chloride salt clusters in the UPLC-MS dataset, comparison of oPLS-DA models using negative ion mode data, comparison of oPLS-DA models using combined negative and positive ion mode data, identification of discriminant features in oPLS-DA classification of APE from stable CF samples using combined positive and negative ion mode data, identification of discriminant features in oPLS-DA classification of pre-APE from stable CF samples using combined positive and negative ion mode data, heat map visualization of stable CF-based z-scores of metabolites, oPLS-DA cross-validated classification plots using combined positive and negative ion mode data, oPLS-DA classification plot for pre-APE and stable CF samples using combined positive and negative mode 
data without splitting the cohort into adult and pediatric subgroups, lactic acid level versus patient age in pre-APE and stable CF patients, positive ion mode MS/MS spectrum of the feature with $m / z 175.1188$, and data analysis workflow (PDF)

\section{AUTHOR INFORMATION}

\section{Corresponding Authors}

*E-mail: astecen@emory.edu. Ph: 404385 4432. Fax: 404385 6447 (A.S.).

*E-mail: facundo.fernandez@chemistry.gatech.edu (F.M.F.). ORCID 1

María Eugenia Monge: 0000-0001-6517-5301

Facundo M. Fernández: 0000-0002-0302-2534

Notes

The authors declare no competing financial interest.

\section{ACKNOWLEDGMENTS}

This study used samples made available by the CF Biospecimen Registry, a part of the Emory + Children's Center for CF and Airways Disease Research, and was supported by philanthropic donations and by a pilot grant within the CF@ LANTA Research Development Program as funded by the Cystic Fibrosis Foundation (MCCART15R0). M.E.M. is a Research Staff member from CONICET (Consejo Nacional de Investigaciones Cientificas y Técnicas, Argentina).

\section{REFERENCES}

(1) Bhatt, J. M. Treatment of pulmonary exacerbations in cystic fibrosis. Eur. Resp. Rev. 2013, 22, 205-216.

(2) Whelan, F. J.; Surette, M. G. Clinical Insights into Pulmonary Exacerbations in Cystic Fibrosis from the Microbiome. What Are We Missing? Ann. Am. Thorac. Soc.Ann Am Thorac Soc 2015, 12, S207S211.

(3) Counterman, A. E.; Hilderbrand, A. E.; Barnes, C. A. S.; Clemmer, D. E. Formation of peptide aggregates during ESI: Size, charge, composition, and contributions to noise. J. Am. Soc. Mass Spectrom. 2001, 12, 1020-1035.

(4) VanDevanter, D. R.; Heltshe, S. L.; Spahr, J.; Beckett, V. V.; Daines, C. L.; Dasenbrook, E. C.; Gibson, R. L.; Jain, R.; Sanders, D. B.; Goss, C. H.; Flume, P. A.; Group, S. S. Rationalizing endpoints for prospective studies of pulmonary exacerbation treatment response in cystic fibrosis. J. Cystic Fibrosis 2017, 16, 607-615.

(5) Rogers, G. B.; Hoffman, L. R.; Johnson, M. W.; Mayer-Hamblett, N.; Schwarze, J.; Carroll, M. P.; Bruce, K. D. Using bacterial biomarkers to identify early indicators of cystic fibrosis pulmonary exacerbation onset. Expert Rev. Mol. Diagn. 2011, 11, 197-206.

(6) Shoki, A. H.; Mayer-Hamblett, N.; Wilcox, P. G.; Sin, D. D.; Quon, B. S. Systematic review of blood biomarkers in cystic fibrosis pulmonary exacerbations. Chest 2013, 144, 1659-1670.

(7) Patti, G. J.; Yanes, O.; Siuzdak, G. Metabolomics: the apogee of the omics trilogy. Nat. Rev. Mol. Cell Biol. 2012, 13, 263-269.

(8) Laguna, T. A.; Reilly, C. S.; Williams, C. B.; Welchlin, C.; Wendt, C. H. Metabolomics analysis identifies novel plasma biomarkers of cystic fibrosis pulmonary exacerbation. Pediatr. Pulmonol. 2015, 50, 869-877.

(9) Quinn, R. A.; Lim, Y. W.; Mak, T. D.; Whiteson, K.; Furlan, M.; Conrad, D.; Rohwer, F.; Dorrestein, P. Metabolomics of pulmonary exacerbations reveals the personalized nature of cystic fibrosis disease. PeerJ 2016, 4, No. e2174.

(10) Rosias, P. P. R.; Dompeling, E.; Hendriks, H. J. E.; Heijnens, J. W. C. M.; Donckerwolcke, R. A. M. G.; Jobsis, Q. Exhaled breath condensate in children: pearls and pitfalls. Pediatr. Allergy Immunol. 2004, 15, 4-19.
(11) Montuschi, P.; Paris, D.; Melck, D.; Lucidi, V.; Ciabattoni, G.; Raia, V.; Calabrese, C.; Bush, A.; Barnes, P. J.; Motta, A. NMR spectroscopy metabolomic profiling of exhaled breath condensate in patients with stable and unstable cystic fibrosis. Thorax 2012, 67, $222-228$.

(12) Zang, X.; Monge, M. E.; McCarty, N. A.; Stecenko, A. A.; Fernández, F. M. Feasibility of Early Detection of Cystic Fibrosis Acute Pulmonary Exacerbations by Exhaled Breath Condensate Metabolomics: A Pilot Study. J. Proteome Res. 2017, 16, 550-558.

(13) Wishart, D. S.; Jewison, T.; Guo, A. C.; Wilson, M.; Knox, C.; Liu, Y.; Djoumbou, Y.; Mandal, R.; Aziat, F.; Dong, E. HMDB 3.0the human metabolome database in 2013. Nucleic Acids Res. 2013, 41, D801-D807.

(14) Smith, C. A.; Maille, G. O.; Want, E. J.; Qin, C.; Trauger, S. A.; Brandon, T. R.; Custodio, D. E.; Abagyan, R.; Siuzdak, G. Metlin. Ther. Drug Monit. 2005, 27, 747-751.

(15) Xia, J.; Sinelnikov, I. V.; Han, B.; Wishart, D. S. MetaboAnalyst 3.0-making metabolomics more meaningful. Nucleic Acids Res. 2015, 43, W251-W257.

(16) Dunn, W. B.; Broadhurst, D.; Begley, P.; Zelena, E.; FrancisMcIntyre, S.; Anderson, N.; Brown, M.; Knowles, J. D.; Halsall, A.; Haselden, J. N.; Nicholls, A. W.; Wilson, I. D.; Kell, D. B.; Goodacre, R. Procedures for large-scale metabolic profiling of serum and plasma using gas chromatography and liquid chromatography coupled to mass spectrometry. Nat. Protoc. 2011, 6, 1060-1083.

(17) McMillan, A.; Renaud, J. B.; Gloor, G. B.; Reid, G.; Sumarah, M. W. Post-acquisition filtering of salt cluster artefacts for LC-MS based human metabolomic studies. J. Cheminf. 2016, 8, 44.

(18) Phan, J.; Gallagher, T.; Oliver, A.; England, W. E.; Whiteson, K. Fermentation products in the cystic fibrosis airways induce aggregation and dormancy-associated expression profiles in a CF clinical isolate of Pseudomonas aeruginosa. FEMS Microbiol. Lett. 2018, 365, fny082.

(19) Twomey, K. B.; Alston, M.; An, S.-Q.; O'Connell, O. J.; McCarthy, Y.; Swarbreck, D.; Febrer, M.; Dow, J. M.; Plant, B. J.; Ryan, R. P. Microbiota and metabolite profiling reveal specific alterations in bacterial community structure and environment in the cystic fibrosis airway during exacerbation. PLoS One 2013, 8, No. e82432.

(20) Wolak, J. E.; Esther, C. R., Jr.; O'Connell, T. M. Metabolomic analysis of bronchoalveolar lavage fluid from cystic fibrosis patients. Biomarkers 2009, 14, 55-60.

(21) Díaz-Pérez, A. L.; Núñez, C.; Meza Carmen, V.; CamposGarcía, J. The expression of the genes involved in leucine catabolism of Pseudomonas aeruginosa is controlled by the transcriptional regulator LiuR and by the CbrAB/Crc system. Res. Microbiol. 2018, 169, 324-334.

(22) Mirković, B.; Murray, M. A.; Lavelle, G. M.; Molloy, K.; Azim, A. A.; Gunaratnam, C.; Healy, F.; Slattery, D.; McNally, P.; Hatch, J.; Wolfgang, M.; Tunney, M. M.; Muhlebach, M. S.; Devery, R.; Greene, C. M.; McElvaney, N. G. The Role of Short-Chain Fatty Acids, Produced by Anaerobic Bacteria, in the Cystic Fibrosis Airway. Am. J. Respir. Crit. Care Med. 2015, 192, 1314-1324.

(23) Rioseras, A. T.; Singh, K. D.; Nowak, N.; Gaugg, M. T.; Bruderer, T.; Zenobi, R.; Sinues, P. M.-L. Real-Time Monitoring of Tricarboxylic Acid Metabolites in Exhaled Breath. Anal. Chem. 2018, 90, 6453-6460.

(24) Tang, X.; Liu, J.; Dong, W.; Li, P.; Li, L.; Lin, C.; Zheng, Y.; Hou, J.; Li, D. The cardioprotective effects of citric Acid and L-malic Acid on myocardial ischemia/reperfusion injury. J. Evidence-Based Complementary Altern. Med. 2013, 2013, 820695.

(25) Koeth, R. A.; Wang, Z.; Levison, B. S.; Buffa, J. A.; Org, E.; Sheehy, B. T.; Britt, E. B.; Fu, X.; Wu, Y.; Li, L.; Smith, J. D.; DiDonato, J. A.; Chen, J.; Li, H.; Wu, G. D.; Lewis, J. D.; Warrier, M.; Brown, J. M.; Krauss, R. M.; Tang, W. H. W.; Bushman, F. D.; Lusis, A. J.; Hazen, S. L. Intestinal microbiota metabolism of L-carnitine, a nutrient in red meat, promotes atherosclerosis. Nat. Med. 2013, 19, 576-585. 
(26) Meadows, J. A.; Wargo, M. J. Carnitine in bacterial physiology and metabolism. Microbiology 2015, 161, 1161-1174.

(27) Strandvik, B. Fatty acid metabolism in cystic fibrosis. Prostaglandins, Leukotrienes Essent. Fatty Acids 2010, 83, 121-129.

(28) Freedman, S. D.; Blanco, P. G.; Zaman, M. M.; Shea, J. C.; Ollero, M.; Hopper, I. K.; Weed, D. A.; Gelrud, A.; Regan, M. M.; Laposata, M.; Alvarez, J. G.; O'Sullivan, B. P. Association of cystic fibrosis with abnormalities in fatty acid metabolism. N. Engl. J. Med. 2004, 350, 560-569.

(29) Kovesi, T. A.; Lehotay, D. C.; Levison, H. Plasma carnitine levels in cystic fibrosis. J. Pediatr. Gastroenterol. Nutr. 1994, 19, 421424.

(30) Carpenedo, R.; Mannaioni, G.; Moroni, F. Oxindole, a sedative tryptophan metabolite, accumulates in blood and brain of rats with acute hepatic failure. J. Neurochem. 1998, 70, 1998-2003.

(31) Puccetti, M.; Paolicelli, G.; Oikonomou, V.; De Luca, A.; Renga, G.; Borghi, M.; Pariano, M.; Stincardini, C.; Scaringi, L.; Giovagnoli, S.; Ricci, M.; Romani, L.; Zelante, T. Towards Targeting the Aryl Hydrocarbon Receptor in Cystic Fibrosis. Mediators Inflammation 2018, 2018, 1601486.

(32) Wetmore, D. R.; Joseloff, E.; Pilewski, J.; Lee, D. P.; Lawton, K. A.; Mitchell, M. W.; Milburn, M. V.; Ryals, J. A.; Guo, L. Metabolomic profiling reveals biochemical pathways and biomarkers associated with pathogenesis in cystic fibrosis cells. J. Biol. Chem. 2010, 285, 30516-30522.

(33) Quinn, R. A.; Phelan, V. V.; Whiteson, K. L.; Garg, N.; Bailey, B. A.; Lim, Y. W.; Conrad, D. J.; Dorrestein, P. C.; Rohwer, F. L. Microbial, host and xenobiotic diversity in the cystic fibrosis sputum metabolome. ISME J. 2016, 10, 1483-1498.

(34) Ayyar, L.; Hsu, J.; Jahoor, F.; Kao, C. The Relationship Of Arginine And Its Metaboites In Exhaled Breath Condensate To Lung Function. Am. J. Respir. Crit. Care Med. 2014, 189, A5645.

(35) Grasemann, H.; Schwiertz, R.; Grasemann, C.; Vester, U.; Racke, K.; Ratjen, F. Decreased systemic bioavailability of L-arginine in patients with cystic fibrosis. Respir. Res. 2006, 7, 87.

(36) Gray, L. R.; Tompkins, S. C.; Taylor, E. B. Regulation of pyruvate metabolism and human disease. Cell. Mol. Life Sci. 2014, 71, $2577-2604$.

(37) Berger, A.; Dohnt, K.; Tielen, P.; Jahn, D.; Becker, J.; Wittmann, C. Robustness and Plasticity of Metabolic Pathway Flux among Uropathogenic Isolates of Pseudomonas aeruginosa. PLoS One 2014, 9, No. e88368.

(38) Li, J.; Greenwood, P. L.; Cockett, N. E.; Hadfield, T. S.; Vuocolo, T.; Byrne, K.; White, J. D.; Tellam, R. L.; Schirra, H. J. Impacts of the Callipyge mutation on ovine plasma metabolites and muscle fibre type. PLoS One 2014, 9, No. e99726. 